\title{
Detection of new sources in IBIS/ISGRI survey data
}

\section{A. Segreto, G. Cusumano, V. La Parola}

INAF, Istituto di Astrofisica Spaziale e Fisica Cosmica, sez. Palermo

Via Ugo La Malfa 153, 90146 Palermo, Italy

E-mail: segreto@ifc.inaf.it

In the 8 years since INTEGRAL has been in orbit many new high energy sources have been discovered with the gamma-ray coded-mask telescope IBIS/ISGRI. Here we report on the preliminary results obtained processing the ISGRI archive available to date with our own developed imaging software.

Comparing our results with the published INTEGRAL catalogues we detect several new sources; we present a preliminary list of those newly discovered source for which we have found a counterpart by cross-correlating their position with catalogs at different wavelengths.

8th INTEGRAL Workshop "The Restless Gamma-ray Universe"

September 27-30 2010

Dublin Castle, Dublin, Ireland 


\section{Introduction}

Two coded mask imagers are currently observing the sky in the hard X-ray domain: the IBIS [1] on board INTEGRAL satellite and the Burst Alert Telescope (BAT) [2] onboard the Swift satellite.

In order to perform a systematic search for new hard X-ray sources in both the ISGRI and BAT survey data, we have developed a dedicated software, the BatImager, which has been designed to generate cleaned all-sky mosaics of a generic coded mask instrument in a very efficient and fast way. The main scientific results and source catalogs obtained analyzing with our software the BAT survey data have been reported in [3] and [4]; here we present the imaging performance of our software applied to the IBIS/ISGRI data and a preliminary sample of the newly detected hard $\mathrm{X}$-ray sources.

\section{The code}

For a detailed description of our software and the method used for skymap reconstruction, we refer reader to [5]; here we underline that, in order to reduce systematic noise and improve the survey sensitivity, we have carefully optimized several aspects, in particular:

- the modeling of the non uniform detector background,

- the computation pixel by pixels of the detection efficiency as a function of time,

- the use of a coded mask model derived from the observed shadows of bright sources,

- the accurate correction of detector pointing misalignment,

- the calibration of the flux attenuation vs. off-axis and energy,

- the computation and subtraction of the illumination patterns of all known sources.

To avoid the problem of angular deformations present in any traditional 2D all-sky projections (e.g. AITOFF), our software accumulates the all-sky mosaics on an HEALPix (Hierarchical Equal Area iso-Latitude Pixelization) [6] spherical grid, particularly suitable to simplify any subsequent skymap analysis. Our all-sky mosaics are subdivided in $\approx 2.9 \cdot 10^{7}$ equal area sky pixels, corresponding to an angular resolution of $\approx 2.5$ arcmin.

In order to verify that the all-sky mosaic maps obtained are suitable for searching new weak sources, two main aspects must be verified: the good removal of the ghosts of bright sources and the good uniformity and statistical distribution of the skymap background.

\subsection{Ghost subtraction}

Due to the periodicity of IBIS mask pattern, very significant side peaks (ghosts) appear in its Point Spread Function (PSF), the most significant located in a square $\left(\approx 10.5^{\circ}\right.$ half side) centered on the source position. If the illumination patterns of all known sources are not properly modeled and subtracted, the systematic residuals in the regions near bright sources could be strong enough to cause the detection of several fake sources.

To demonstrate the accuracy level obtained with our software in ghost subtraction, we show in Fig. 1 the $20-60 \mathrm{keV}$ significance skymap of $25^{\circ} \times 25^{\circ}$ regions around the four brightest sources; as it is clearly seen, there is no evidence of the cross-shaped or circular-shaped structures that are the typical characteristics of not well subtracted ghosts (see, e.g., [7]). 

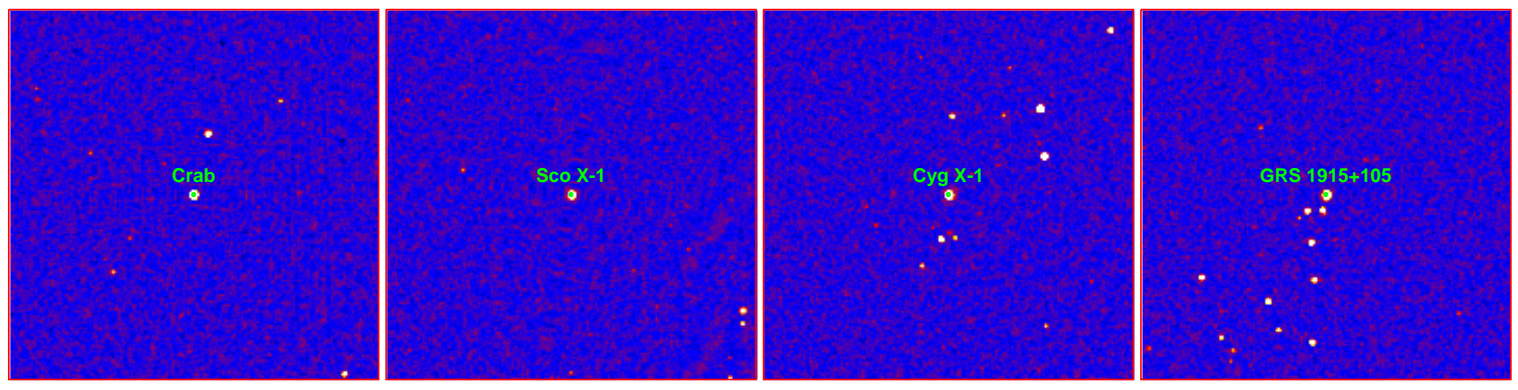

0

10

Figure 1 The $20-60 \mathrm{keV}$ significance skymap regions $\left(25^{\circ} \times 25^{\circ}\right)$ around the four brightest

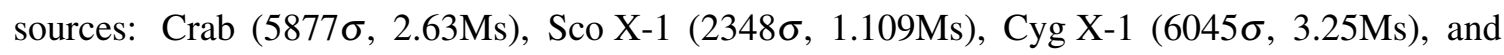
GRS $1915+105(3105 \sigma, 5.61 \mathrm{Ms})$. The detection significance and the on-source corrected observing time, are indicated in brackets. There is no evidence of the typical structures generated by an imperfect subtraction of PSF ghosts.

\subsection{Statistical distribution of mosaics noise}

Due to residual systematics, the mosaic background can present large scale spatial structures that, although of small intensity, alter the statistical significance of the source detections.

To remove these structures, we measure the local statistic (mean value and variance) of the skymap (in regions much larger than the PSF and excluding sources), and rescale the skymaps values accordingly; in this way we obtain a cleaned sky image characterized by a uniform background with a $\mathrm{S} / \mathrm{N}$ distribution that (excluding the contribution of sources) is consistent with a Gaussian with zero mean and unitary standard deviation; on these skymaps it is then possible to perform the statistically reliable detection of weak sources even in sky region with deep exposure. As an example, in Figure 2 we show the 20-60 keV significance skymap near the Galactic Center (16.24 Ms) and its statistical distribution.
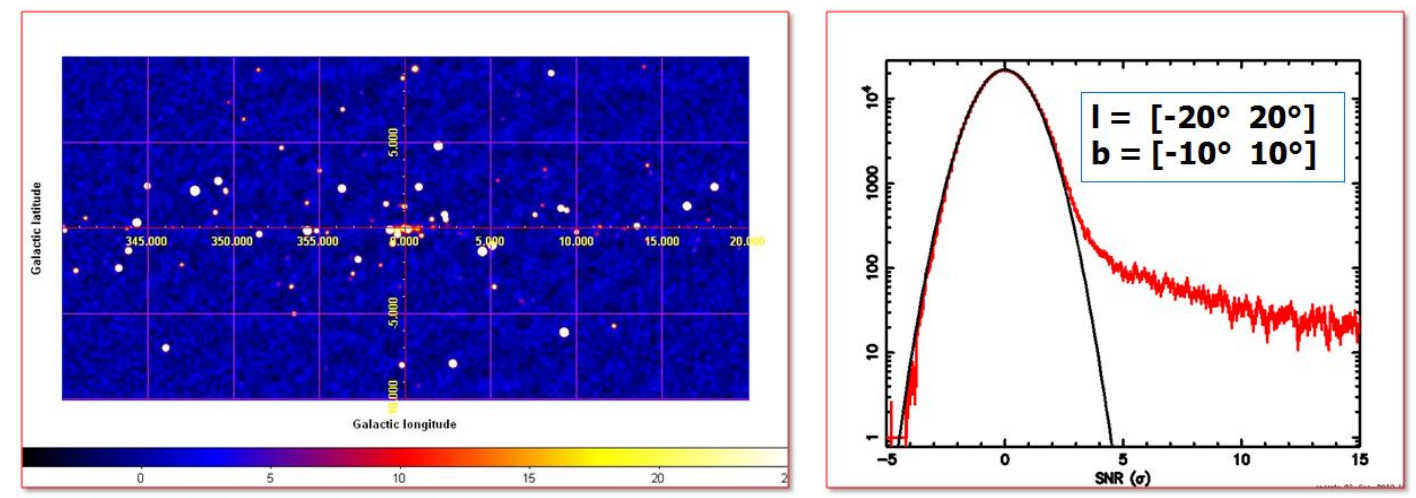

Figure 2 Left: IBIS/ISGRI 20-60 keV significance map near the Galactic Plane region (the exposure at the Galactic Center is $\approx 16.24 \mathrm{Ms}$ ); Right: histogram of significance values in the Galactic Center Plane region. The continuous line is a Gaussian with zero mean and unitary std. dev. 


\section{Search for Transient sources}

X-ray transients which are active during a small fraction of the observing time, may not be detectable in mosaics accumulated using all the available data. To perform a systematic search for these sources we generate a light curve for every pixel in the all-sky mosaics, and compute a few parameters that could indicate the presence of a transient source in that position, like e.g:

- the total standard deviation of the $\mathrm{S} / \mathrm{N}$ lightcurve

- the best $\mathrm{S} / \mathrm{N}$ obtainable integrating the light-curves in sub-intervals of any duration

We then analyze the maps of these parameters searching for values not consistent with random fluctuations.

Of course, because of the non-uniform exposure and the different number of trials for each lightcurve, the statistical distribution of values in these maps is not gaussian and the shape and width of the distribution depend on the pixel position; pixels within a few degrees radius however have the same statistical distribution as they have very similar exposure and same number of lightcurve bins. For each source candidate we then accumulate the histogram of values in a small region centered on the pixel and fit it with an analytical function which is then used to compute the probability that the observed excess is due to a random fluctuation.

As an example of sources detected in this way, we show the case of the High Mass X-ray Binaries 2S 1553-542 and XTE J1543-568; these sources are not detected in the 20-60 keV significance mosaic accumulated using all the IBIS survey data publicly available to date (Fig. 3 left), but their presence is clearly visible in a sky-map where the value in each pixel represents the total standard deviation of the S/N light curve of that pixel (Fig. 3 center).

The lightcurves of these two sources (Fig. 3 right) clearly show their transient nature; in the short time intervals indicated, they are detected with a significance $>9 \sigma$. The ISGRI detection of 2S 1553-542 has never been previously reported.

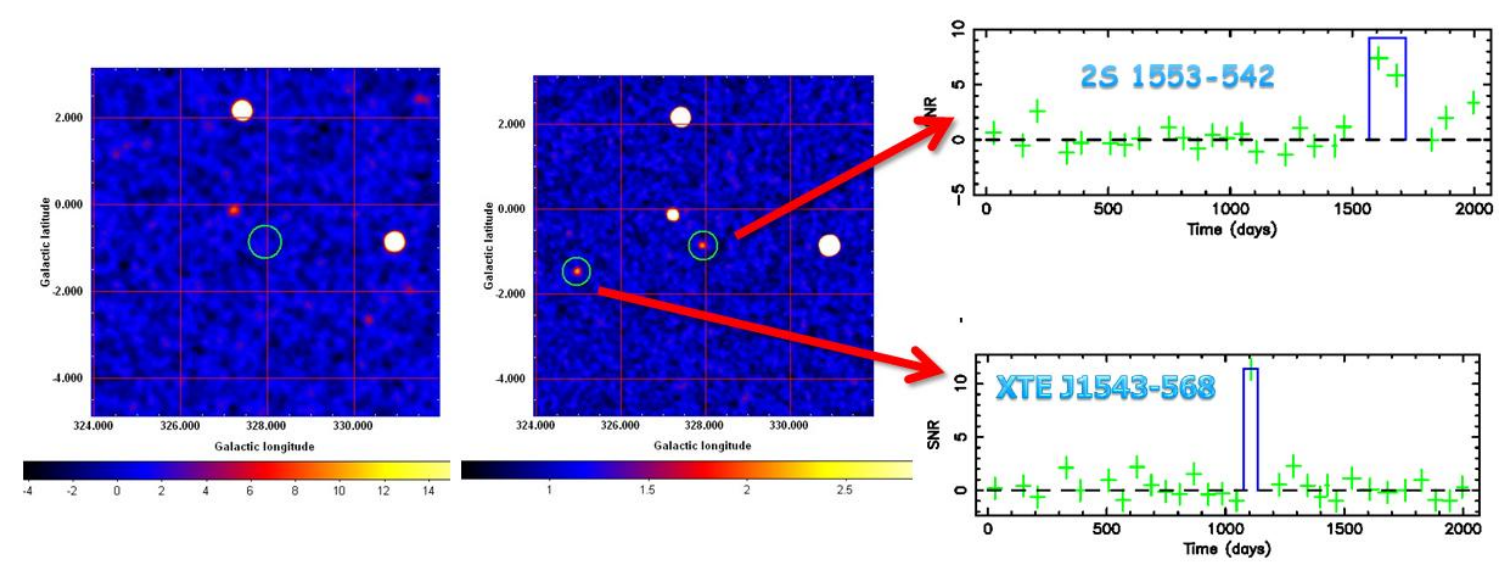

Figure 3 Left: a region of the IBIS/ISGRI significance skymap (20-60 keV, total exposure $\approx 4.5 \mathrm{Ms}$ ); Center: the same sky region where the value in each pixel is the standard deviation of the S/N lightcurve of that pixel; Right: the S/N lightcurves of 2S 1553-542 (first detection reported here) and XTE J1543-568, with indicated the time intervals of best detection. 


\section{Survey analysis and a sample of the newly detected ISGRI sources}

We analyzed the IBIS/ISGRI survey data publicly available to date (for a net observing time of $\approx 154 \mathrm{Ms}$ ) generating the all-sky mosaics in several energy ranges: $15-20 \mathrm{keV}, 20-60 \mathrm{keV}$, 60-100 keV, 20-100 keV, 15-100 keV.

We have then cross-correlated the list of all the sources detected with a significance level $>5 \sigma$ in our mosaics with the the 4th INTEGRAL/IBIS catalog [8], obtaining a reduced list of 156 sources not listed there; among these, 17 have already been reported in [9] while the remaining 139 sources are new detections for the ISGRI instrument.

These new detections are partly due to the additional data we have used and partly due to the new techniques we have implemented; the full catalog and its analysis will be published in a forthcoming paper, here we only give a preliminary list of associations obtained by cross-correlating the list of the new detected sources with other catalogs.

To perform the cross-correlation we have preliminary measured the source localization error in our skymaps by using the offsets of known sources from their nominal position; we have obtained a $90 \%$ error radius $R_{90}=3.1$ arcmin for $5 \sigma$ detections and verified that, for weak sources, it is inversely proportional to the source significance.

For each possible association we have also computed the probability of random association as given by

$$
P_{\text {rand }}=1-\exp \left(-\pi v d^{2}\right)
$$

where $\mathrm{d}$ is the distance between the ISGRI detected source and the associated candidate, and $v$ the local density (computed in a disk of a few degree radius centered on the candidate) of objects in the catalog considered for association.

A preliminary list of the likely optical associations we have found is given in Tab. 1, while in Tab. 2 we list associations with unidentified BAT sources [4] and in Tab. 3 associations with FERMI sources.

\section{References}

[1] Ubertini, P., Lebrun, F., Di Cocco, G., et al., IBIS: The Imager on-board INTEGRAL A\&A, 411, L131 (2003)

[2] S. D. Barthelmy, L. M. Barbier, J. R. Cummings, et al., The Burst Alert Telescope (BAT) on the SWIFT Midex Mission Space Science Reviews, 1204, 143 (2005).

[3] G. Cusumano, V. La Parola, A. Segreto, et al., The Palermo Swift-BAT hard X-ray catalogue. II. Results after 39 months of sky survey A\&A 510, A48 (2010)

[4] G. Cusumano, V. La Parola, A. Segreto, et al., Palermo Swift-BAT hard X-ray catalogue III. Results after 54 months of sky survey, A\&A 524, A64 (2010)

[5] A. Segreto, G. Cusumano, C. Ferrigno, et al., The Palermo Swift-BAT hard X-ray catalogue. I. Methodology, A\&A 510, A47 (2010)

[6] Górski K. M., Hivon, E., Banday, et al., HEALPix: A Framework for High-Resolution Discretization and Fast Analysis of Data Distributed on the Sphere, ApJ 622, 759 (2005) 
Table 1. A preliminary list of the new ISGRI detected hard X-ray sources for which we have found a likely optical counterpart; for each source we indicate its best detection significance, the angular distance (in arcmin) to the ISGRI position, the $90 \%$ error radius and the probability of random association.

\begin{tabular}{rllrrrr}
\hline \hline index & \multicolumn{1}{c}{ Name } & \multicolumn{1}{c}{ Type } & S/N $(\sigma)$ & Offset $\left({ }^{\prime}\right)$ & $\mathrm{R}_{90}\left({ }^{\prime}\right)$ & $\mathrm{P}_{\text {rand }}(\%)$ \\
\hline 1 & QSO B2344+514 & BLLac & 6.7 & 1.7 & 2.3 & 0.6 \\
2 & V* V2306 Cyg & CataclyV* & 7.2 & 0.5 & 2.1 & 2.1 \\
3 & SNR G054.1+00.3 & SNR & 6.0 & 1.6 & 2.6 & 2.9 \\
4 & 2MASX J05081967+1721483 & Sy2 & 6.3 & 1.2 & 2.5 & 0.8 \\
5 & 2MASX J18452628+7211008 & Sy2 & 5.6 & 2.5 & 2.8 & 1.3 \\
6 & RX J2015.6+3711 & CataclyV* & 5.5 & 2.6 & 2.8 & 2.4 \\
7 & ESO 139-12 & Sy2 & 5.6 & 1.8 & 2.7 & 0.3 \\
8 & PSR B1802+03 & Pulsar & 5.2 & 3.1 & 3.0 & 0.4 \\
9 & RORF 1714-336 & BLLac & 5.6 & 1.8 & 2.8 & 1.0 \\
10 & ICRF J063546.5-751616 & Sy1 & 5.3 & 2.9 & 2.9 & 1.1 \\
11 & 2MASX J01344565-0430134 & Sy1 & 5.2 & 0.9 & 3.0 & 1.1 \\
12 & 2MASX J02153739-1259298 & Sy2 & 5.1 & 0.9 & 3.0 & 1.6 \\
\hline
\end{tabular}

Table 2. Likely associations of new ISGRI detected sources with unidentified BAT sources

\begin{tabular}{rcrrrrrrr}
\hline \hline index & ISGRI Name & RA $\left(^{\circ}\right)$ & Dec $\left(^{\circ}\right)$ & S/N $(\sigma)$ & $R_{90}\left({ }^{\prime}\right)$ & BAT Name & Offset $\left({ }^{\prime}\right)$ & P $_{\text {rand }}(\%)$ \\
\hline 1 & IGR J16320-5620 & 248.000 & -56.339 & 5.0 & 3.1 & PBC J1631.9-5616 & 4.0 & 0.4 \\
2 & IGR J16182-5408 & 244.560 & -54.140 & 5.4 & 2.9 & PBC J1618.1-5405 & 2.7 & 0.1 \\
3 & IGR J17014-4304 & 255.360 & -43.081 & 6.0 & 2.6 & PBC J1701.6-4307 & 3.4 & 0.6 \\
4 & IGR J16491-1738 & 252.295 & -17.647 & 6.1 & 2.5 & PBC J1649.3-1739 & 2.4 & 0.1 \\
5 & IGR J05153+1855 & 78.837 & 18.919 & 9.3 & 1.7 & PBC J0515.3+1856 & 1.2 & 0.0 \\
6 & IGR J11276-5323 & 171.924 & -53.395 & 5.4 & 2.9 & PBC J1127.7-5320 & 3.2 & 0.2 \\
7 & IGR J05163+1927 & 79.096 & 19.464 & 5.3 & 2.9 & PBC J0516.3+1927 & 1.0 & 0.0 \\
\hline
\end{tabular}

Table 3. Likely associations of new ISGRI detected sources with FERMI sources

\begin{tabular}{rcccrccrc}
\hline \hline index & ISGRI Name & RA $\left(^{\circ}\right)$ & Dec $\left({ }^{\circ}\right)$ & $\mathrm{S} / \mathrm{N}(\sigma)$ & $\mathrm{R}_{90}\left({ }^{\prime}\right)$ & FERMI Name & Offset $\left({ }^{\prime}\right)$ & $\mathrm{P}_{\text {rand }}(\%)$ \\
\hline 1 & IGR J23472+5143 & 356.809 & 51.719 & 6.7 & 2.3 & 1FGL J2347.1+5142 & 1.3 & 0.0 \\
2 & IGR J17441-2928 & 266.029 & -29.481 & 29.8 & 0.5 & 1FGL J1744.0-2931c & 3.4 & 0.8 \\
3 & IGR J18133-1246 & 273.339 & -12.780 & 5.2 & 3.0 & 1FGL J1813.3-1246 & 1.0 & 0.0 \\
4 & IGR J17478-2818 & 266.974 & -28.316 & 10.9 & 1.4 & 1FGL J1747.6-2820c & 3.2 & 0.6 \\
5 & IGR J17471-2954 & 266.796 & -29.907 & 7.2 & 2.2 & 1FGL J1747.2-2958 & 3.8 & 0.9 \\
6 & IGR J17142-3829 & 258.574 & -38.499 & 5.7 & 2.7 & 1FGL J1714.5-3830c & 3.4 & 0.7 \\
7 & IGR J16258-2527 & 246.451 & -25.462 & 5.3 & 2.9 & 1FGL J1625.7-2524 & 3.0 & 0.3 \\
\hline
\end{tabular}


[7] R. Krivonos, M. Revnivtsev, S. Tsygankov, et al., INTEGRAL All-Sky Survey: Deep inside Galaxy and beyond, in proceedings of The Extreme sky: Sampling the Universe above $10 \mathrm{keV}$, PoS (extremesky2009) 004 .

[8] A. J. Bird et al., 4th INTEGRAL/IBIS sky survey catalogue, ApJS 186, 1, (2010)

[9] R. Krivonos et al., INTEGRAL/IBIS 7-year All-Sky Hard X-Ray Survey, A\&A, 523 A61 (2010) 